\title{
Comparisons of Controller Performance for Small-Scale Vertical Axis Wind Turbines*
}

\author{
Aykut O Onol, Ahmet Onat, Serhat Yesilyurt, Senior Member, IEEE
}

\begin{abstract}
Small-scale vertical axis wind turbines (VAWTs) are attractive for portable power generation. Controller performance is very important in rapidly varying gusty winds commonly observed in urban and rural areas. In this paper, a hill-climb searching (HSC) maximum power point tracking (MPPT), an energy-maximizing model predictive control (MPC) and a simple nonlinear control (SNC) as an MPC surrogate are presented. The control algorithms are tested through a software-only electromechanical model and with hardware-in-theloop test-bed that includes electromechanical and power electronics components. Effects of power coefficient oscillations on dynamic performance are investigated. Results show that proposed controllers perform satisfactorily for wind gust and real wind profiles; the SNC serves as a viable surrogate for the MPC; the model-free, wind speed sensorless MPPT is favorable for small-scale applications; and power coefficient oscillations do not have a significant impact on the dynamic performance of the controllers.
\end{abstract}

\section{INTRODUCTION}

Large-scale wind power plants are optimized for steady winds [1]. Omnidirectional vertical axis wind turbines (VAWTs) are advantageous in gusty, turbulent winds with rapidly changing direction such as surface winds [2], and favorable in urban areas, e.g., on top of a building, as well as in rural areas away from the integrated grid systems [2-6].

Control for large-scale wind turbines combine multiple objectives such as maximization of energy, reduction of mechanical loads on tower and blades, and smoothing of power gradients, and the control variables are generator torque, blade pitch angle, and yaw angle [7,8]. Small-scale VAWTs may avoid mechanical limitations such as blade bending and capture energy from extreme winds; hence, energy maximization subject to electrical system becomes the main objective.

Maximum power point tracking (MPPT) is popular for

${ }^{*}$ Research supported by Sabanci University Internal Research Grant Program, SU-IRG-985.

Aykut O. Onol is former MS graduate from the Mechatronics Program in Sabanci University and currently a $\mathrm{PhD}$ student in the Computer Engineering Department in Northeastern University onol.a@husky.neu.edu

Ahmet Onat is an Associate Professor at the Mechatronics Program in the Faculty of Engineering and Natural Sciences in Sabanci University onat@sabanciuniv.edu

Serhat Yesilyurt is a Professor at the Mechatronics Program in Faculty of Engineering and Natural Sciences in Sabanci University, and currently with the Mechanical Engineering Department in University of Michigan as a visiting scholar syesilyurt@sabanciuniv.edu coping with various unsteady effects in renewable energy systems [9]. MPPT control techniques for HAWTs are classified into four categories [10]: tip-speed ratio control, optimal torque control, power signal feedback control, and hill-climb searching (HCS) control, which does not require a turbine model or wind speed measurement. Koutroulis and Kalaitzakis [11] propose a generic HCS MPPT technique to maximize the power output of wind energy conversion with $10-50 \%$ increase in the power output. Nevertheless, maximizing the instantaneous power does not guarantee maximum energy generation [12].

For large-scale HAWTs, model predictive control (MPC) is proven to maximize energy efficiency [13], load reduction [14], improvement of power quality [15] and handling of additional constraints [16]. However, the cost of a prediction system and computational power requirements may be restrictive to use such an advanced technique for small-scale applications. Moreover, uncertainties in the wind speed may hinder the performance of the MPC. Nonetheless, the response of an MPC that maximizes the energy generation subject to electrical constraints of a VAWT system for arbitrary wind conditions, such as in [12], is insightful for the design of a simpler controller.

In this study, we present a model for a small-scale VAWT system that consists of a three-straight-bladed rotor, a permanent magnet synchronous generator (PMSG), a full-bridge rectifier and a pure-resistive load. The model includes the realistic aerodynamics of the rotor based on [17] and a simplified DC model for the PMSG - rectifier - load structure based on $[18,19]$. The model is used to design and evaluate an HCS MPPT algorithm, an MPC to maximize the energy generation subject to electrical constraints. A simple nonlinear control (SNC) is designed as a surrogate for the MPC. Hardware-in-the-loop test-bed developed in previous work $[18,19]$ is used to test the controllers. Lastly, effects of power coefficient oscillations are investigated.

\section{Methodology}

\section{A. Rotor Dynamics}

The rate of change of the angular velocity of the rotor, $\omega$, is obtained from the conservation of the angular momentum:

$$
\frac{d \omega}{d t}=\frac{T_{\text {wind }}-T_{g e n}-T_{f}}{J}
$$

where $J$ is the inertia of the rotor, $T_{\text {wind }}$ wind torque on the rotor, $T_{\text {gen }}$ the generator torque and $T_{f}$ the friction torque on the shaft. 
The mechanical power of a Darrieus rotor, $P_{\text {wind }}$, is calculated from the wind velocity, $U$, the air density, $\rho$, the rotor radius, $R$, the rotor height, $L$, and the tip-speed ratio (i.e., $\lambda=\omega R / U)$ as $P_{\text {wind }}=C_{P}(\lambda, t) \rho L R U^{3}$. Thus, if the $\lambda-C_{P}(\lambda, t)$ relation of the rotor is known, $T_{\text {wind }}$ can be obtained as below:

$$
T_{\text {wind }}=\frac{P_{\text {wind }}}{\omega}=\frac{C_{P}(\lambda, t) \rho L R U^{3}}{\omega}
$$

For a three-straight-bladed rotor, the instantaneous power coefficient has a sinusoidal pattern [20,17], which is composed of a steady component, $C_{P, a v g}(\lambda)$, and oscillations with an amplitude of $C_{P, \text { amp }}(\lambda)$. The frequency of the oscillations is exactly $3 \omega$ consistently with the three-bladed structure, and typically the $C_{P, a m p}$ increases with the $\lambda$. Thus, the unsteady power coefficient, $C_{P}(\lambda, t)$, can be expressed as:

$$
C_{P}(\lambda, t)=C_{P, \text { avg }}(\lambda)+C_{P, \text { amp }}(\lambda) \sin (3 \omega t)
$$

In this study, we use the $\lambda-C_{P, a v g}$ curve shown in Fig. 1a to estimate the steady part and the $\lambda-C_{P, \text { amp }}$ curve shown in Fig. $1 \mathrm{~b}$ to estimate the amplitude of the oscillations from transient computational fluid dynamics (CFD) simulations presented in [17]. Similar results for the unsteady power coefficient of VAWTs with straight blades are reported in literature [21].

In electromechanical and HIL simulations, the rotor dynamics in (1) is numerically solved by the forward Euler method with time steps of $1 \mathrm{~ms}$, and the $\lambda-C_{P, a v g}$ curve shown in Fig. 1a is used to estimate the $T_{\text {wind }}, C_{P}$ oscillations are only considered in Section III.C.
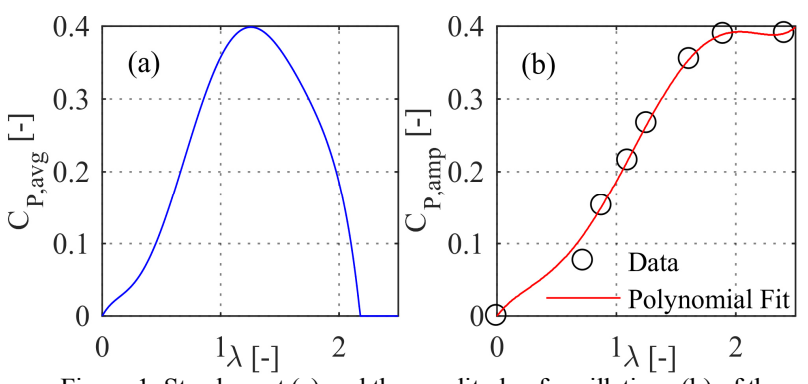

Figure 1: Steady part (a) and the amplitude of oscillations (b) of the $C_{P}$ vs $\lambda$.

\section{B. Electromechanical Model}

In the VAWT system, a PMSG is used for electromechanical energy conversion, and its output is connected to a pure-resistive electronic-load via a passive full-bridge rectifier. The generator torque, $T_{g e n}$, is the product of the load current, $I_{L}$, and the torque constant, $K_{t}$, i.e., $T_{g e n}=K_{t} I_{L}$, and the back electromotive force (EMF) voltage, $E_{L N}$, is the product of the flux, $\varphi_{s}$, the number of pole pairs, $p$, and the angular velocity, $\omega$, i.e., $E_{L N}=\varphi s p \omega$.

The load voltage, $V_{L}$, is correlated with the rotor velocity when the $I_{L}$ is zero; however, the $V_{L}$ drops as the $I_{L}$ increases due to losses. Thus, a power loss model is required to estimate the power output, $P_{\text {gen }}$, and the $V_{L}$ for given $\omega$ and $I_{L}$.

The electronic-load is operated in the galvanostatic mode as a current sink. Thus, the three-phase PMSG - rectifier load model can be simplified into an equivalent DC model with back EMF voltage $E_{d c}=3 \sqrt{6} E_{L N} / \pi$, stator inductance $L_{d c}=18 L_{S} / \pi^{2}$, and stator resistance $R_{S}=18 R_{S} / \pi^{2}$, where $L_{S}$ and
$R_{S}$ are the phase inductance and resistance, respectively. In addition, the armature reaction in the PMSG and the overlapping currents in the rectifier lead to a resistance term, $R_{\text {over }}$, given by:

$$
R_{\text {over }}=3 L_{S} p \omega / \pi
$$

Lastly, the full-bridge rectifier introduces a voltage drop twice the diode threshold voltage, $V_{t h}$, since two diodes commute for each phase. The constant parameters that are used in the rotor dynamics and the electromechanical model are summarized in Table 1.

TABLE I. ELECTROMECHANICAL MODEL PARAMETERS

\begin{tabular}{|c|c|c|}
\hline Parameter & Value & Unit \\
\hline$\rho$ & 1.205 & $\mathrm{~kg} / \mathrm{m}^{3}$ \\
\hline$L$ & 1 & $\mathrm{~m}$ \\
\hline$R$ & 0.5 & $\mathrm{~m}$ \\
\hline$J$ & 2 & $\mathrm{~kg}-\mathrm{m}^{2}$ \\
\hline$p$ & 6 & - \\
\hline$\varphi_{s}$ & 0.1060 & $\mathrm{~V}-\mathrm{s} / \mathrm{rad}$ \\
\hline$L_{S}$ & 3.3000 & $\mathrm{mH}$ \\
\hline$R_{S}$ & 1.5500 & $\Omega$ \\
\hline$V_{t h}$ & 0.7700 & $\mathrm{~V}$ \\
\hline$K_{t}$ & 1.4877 & $\mathrm{~N}-\mathrm{m} / \mathrm{A}$ \\
\hline
\end{tabular}

Although the electronic-load is pure-resistive, the real power, $P$, is not equal to the apparent power, i.e., $S=E_{d c} I_{L}$, due to the reactive power caused by the stator inductance, i.e., $Q=L_{d c} p \omega I_{L}^{2}$, and it is given by:

$$
P=\sqrt{S^{2}-Q^{2}}=\sqrt{\left(E_{d c} I_{L}\right)^{2}-\left(L_{d c} p \omega I_{L}^{2}\right)^{2}}
$$

Moreover, the stator resistance and the voltage drop associated with the rectifier cause power losses $P_{S}$ and $P_{R}$, respectively, i.e., $P_{S}=R_{d c} I_{L}^{2}$ and $P_{R}=R_{\text {over }} I_{L}^{2}+2 V_{t h} I_{L}$. Thus, the net power output is obtained in terms of the $I_{L}$ and $\omega$ as follows:

$$
P_{g e n}=\sqrt{\left(E_{d c} I_{L}\right)^{2}-\left(L_{d c} p \omega I_{L}^{2}\right)^{2}}-R_{d c} I_{L}^{2}-R_{o v e r} I_{L}^{2}-2 V_{t h} I_{L}
$$

\section{Hardware-in-the-loop Setup}

We use the HIL test-bed presented in [18, 19], which comprises of a PC, an electrical motor, a gearbox, a PMSG, a full-bridge rectifier, and a programmable electronic-load. The $\mathrm{PC}$, which operates the software for the emulation of the dynamics and the control, and the hardware components are interconnected through a dSPACE interface. The motor torque is calculated from the $T_{\text {wind }}$ (based on the $\lambda-C_{P, a v g}$ curve shown in Fig. 1a), the $T_{g e n}$, the $T_{f}$, and the gear ratio. The $T_{f}$ is estimated as a function of $\omega$ from HIL experiments as follows:

$$
T_{f}(\omega)=-1.417 \times 10^{-8} \omega^{2}+1.327 \times 10^{-4} \omega+0.175
$$

The disturbance torques caused by the friction in the drivetrain and the cogging torque are overcome by using a disturbance torque compensator comprising of a virtual plant and a proportional-integral controller.

\section{Maximum Power Point Tracking}

The hill-climb searching (HCS) MPPT manipulates the $I_{L}$ based on the $V_{L}$ measurement at time step, $k$. The $I_{L}$ and $V_{L}$ at time step, $k+1$, can be expressed in terms of the change of the $I_{L}$ and $V_{L}, \Delta I_{L}$ and $\Delta V_{L}$, as follows:

$$
\{I, V\}_{L, k+1}=\{I, V\}_{L, k}+\Delta\{I, V\}_{L, k}
$$


Additionally, the $\Delta V_{L}$ can be defined as the product of the partial derivative of the $V_{L}$ with respect to the $I_{L}$ and the $\Delta I_{L}$ :

$$
\Delta V_{L, k}=\left.\frac{\partial V_{L}}{\partial I_{L}}\right|_{k, \omega_{k}} \Delta I_{L, k}
$$

The $\partial V_{L} / \partial I_{L}$ at time step $k$, when the $\omega$ does not vary considerably, is a negative constant due to Ohm's law, i.e., $\partial V_{L} / \partial I_{L}=-\kappa$, and $\kappa>0$, so $V_{L, k+1}$ can be rewritten as:

$$
V_{L, k+1}=V_{L, k}-\kappa \Delta I_{L, k}
$$

The change in the generator power, $P_{\text {gen }}$, reads:

$$
\Delta P_{g e n, k+1}=P_{g e n, k+1}-P_{g e n, k}=\left(V_{L, k+1} I_{L, k+1}\right)-\left(V_{L, k} I_{L, k}\right)
$$

Substituting (8) and (10) in (11) yields:

$$
\Delta P_{g e n, k}=V_{L, k} \Delta I_{L, k}-\kappa I_{L, k} \Delta I_{L, k}-\kappa\left(\Delta I_{L, k}\right)^{2}
$$

The variation in $P_{\text {gen }}$ with respect to the $I_{L}$ must be zero at the maximum power point (MPP), then:

$$
\begin{gathered}
\left.\frac{\partial P_{g e n}}{\partial I_{L}}\right|_{k} \approx \frac{\Delta P_{g e n, k}}{\Delta I_{L, k}}=V_{L, k}-\kappa\left(I_{L, k}+\Delta I_{L, k}\right)=0 \\
\Rightarrow \kappa I_{L, k+1}=V_{L, k}
\end{gathered}
$$

By subtracting $\kappa I_{L, k}=V_{L, k-1}$ from both sides, we obtain:

$$
\kappa\left(I_{L, k+1}-I_{L, k}\right)=V_{L, k}-V_{L, k-1}
$$

From (8) and (10), we have:

$$
\Delta I_{L, k+1}=\Delta V_{L, k} / \kappa
$$

Therefore, the MPPT algorithm modifies the $I_{L}$ proportionally to the $\Delta V_{L}$ through a gain $K$, i.e., $\Delta I_{L, k}=K \Delta V_{L, k}$. The $K$ is selected as 0.2 through a parametric study, and the sampling period, $T_{S}$, is set to $0.1 \mathrm{~s}$.

\section{E. Model Predictive Control}

The goal of the MPC is to find the optimal $I_{L}$ trajectory that maximizes the energy generation for a specified finite prediction-horizon subject to the voltage and current constraints for a measured wind velocity.

The cost function is composed of three terms. The first term, $\Phi_{E}$, is associated with the objective of energy maximization:

$$
\Phi_{E}=-\sum_{i=k}^{k+N-1} P_{g e n, i} T_{S}
$$

where $N$ is the length of the prediction horizon. The second and third terms, $\Phi_{V}$ and $\Phi_{I}$, are penalties for the violation of the voltage and current limits, respectively:

$$
\Phi_{\{V, I\}}=T_{S} \sum_{i=k}^{k+N-1} R\left(\{V, I\}_{L, i}-\{V, I\}_{L, \max }\right)+R\left(-\{V, I\}_{L, i}\right)
$$

where $V_{L, \max }$ and $I_{L, \max }$ are the maximum voltage and current limits that are $60 \mathrm{~V}$ and $15 \mathrm{~A}$, respectively, and $R$ is the ramp function, $R(x)=\max (x, 0)$.

Consequently, the optimization problem is defined as:

$$
\begin{aligned}
& \min _{I_{L, k, \ldots, k+N-1}} w_{1} \Phi_{E}+w_{2}\left(\Phi_{V}+\Phi_{I}\right) \\
& \text { subject to } 0 \leq V_{L} \leq V_{L, \text { max }} \text { and } 0 \leq I_{L} \leq I_{L, \max }
\end{aligned}
$$

where $w_{1}$ and $w_{2}$ are the weights on the objectives of maximizing the energy output and penalizing constraint violations, and they are selected as 1 and $10^{6}$, respectively.

The quasi-newton algorithm of the unconstrained nonlinear programming solver of Matlab (i.e., fminunc) is employed in the optimization procedure. The values of the $T_{S}$ and $N$ are selected as $1 \mathrm{~s}$ and 10 considering the dynamics of the system and the computational burden.

\section{F. Surrogate Control for MPC}

The $\omega, I_{L}, V_{L}$ and $P_{\text {gen }}$ responses of the MPC obtained from an electromechanical simulation for a step wind profile are shown in Fig. 2. When the $V_{L}$ is too low with respect to its reference value (e.g., for $t<2 \mathrm{~s}$ ), MPC algorithm sets the $I_{L}$ to zero. Similarly, when the $V_{L}$ is too high (e.g., for $60<t<$ $65 \mathrm{~s}), I_{L}$ is set to its maximum. The controller adjusts the $I_{L}$ such that the $V_{L}$ converges to its reference (e.g., for $2<t<10$ s) when the $V_{L}$ is sufficiently close to the reference value.

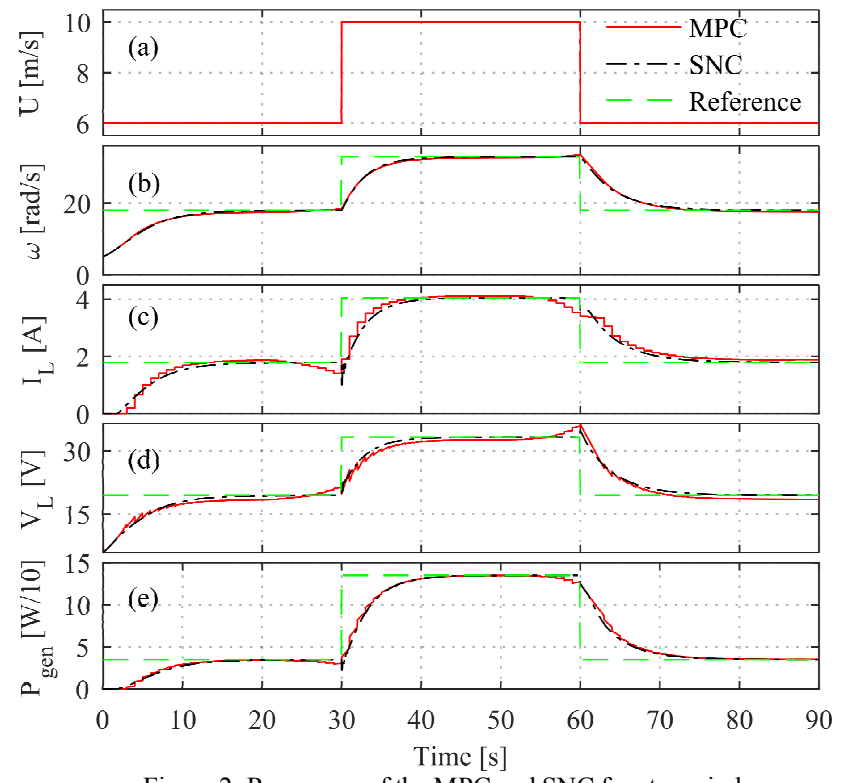

Figure 2: Responses of the MPC and SNC for step wind.

A simple nonlinear control is defined as a piecewise function of the $V_{L}$ to mimic the behavior of the MPC. When the $V_{L}$ is below a lower limit, $V_{L, L}, I_{L}=0$, and when the $V_{L}$ is above an upper limit, $V_{L, U}, I_{L}$ is set to maximum, which is twice the reference value, $I_{L, r e f}$. Between the lower and upper limits, a proportional control is employed to drive the $V_{L}$ to its reference value, $V_{L, r e f}$. as follows:

$$
I_{L, k}=\left\{\begin{array}{cl}
0 & \text { if } V_{L, k} \leq V_{L, L, k} \\
I_{L, r e f, k}-\frac{I_{L, r e f, k} K_{P} e_{k}}{V_{L, r e f, k}-V_{L, L, k}}, & \text { if } \mathrm{V}_{L, L, k}<V_{L, k} \leq V_{L, r e f, k} \\
I_{L, r e f, k}+\frac{I_{L, r e f, k} K_{P} e_{k}}{V_{L, r e f, k}-V_{L, U, k}}, & \text { if } \mathrm{V}_{L, r e f, k}<V_{L, k}<V_{L, U, k} \\
2 I_{L, r e f, k}, & \text { if } \mathrm{V}_{L, k} \geq V_{L, U, k}
\end{array}\right.
$$

where $e$ is the deviation of the voltage from the reference (i.e., $e=V_{L, r e f}-V_{L}$ ) and $K_{P}$ is the proportional gain.

The reference values $P_{\text {gen,ref }}$ and $V_{L, \text { ref }}$ for a given $U$ are obtained by polynomial fits to the maximum power and the 
corresponding $V_{L}$ data points obtained from simulations for a range of steady wind velocities, i.e., $3 \leq U \leq 18 \mathrm{~m} / \mathrm{s}$. The reference value for the current is obtained by $I_{L, r e f}=$ $P_{\text {gen,ref }} / V_{L, r e f}$.

The values of $V_{L, L}$ and $V_{L, U}$ for a given $U$ are obtained from the $V_{L}-C_{P g e n}$ curve, where $C_{P g e n}$ is the generator power coefficient (i.e., $C_{P g e n}=P_{g e n} / \rho L R U^{3}$ ), as follows. For a given level of the $C_{P g e n}, \gamma C_{P g e n}, V_{L, L}$ and $V_{L, U}$ are defined as the lower and upper bounds of the $V_{L}$ for which the $C_{P g e n}$ is greater than $\gamma C_{P g e n}$, as shown in Fig. 3. A parametric study shows that the energy output enhances as the $K_{P}$ increases, however $K_{P}>1$ causes negative $I_{L}$ values; therefore, $K_{P}$ is selected as 1. While, the optimal value for $\gamma$ is obtained as $70 \%$, and the $T_{S}$ for the SNC is $0.1 \mathrm{~s}$. The response of the resulting SNC design mimics the response of the MPC for the step wind profile as shown in Fig. 2.

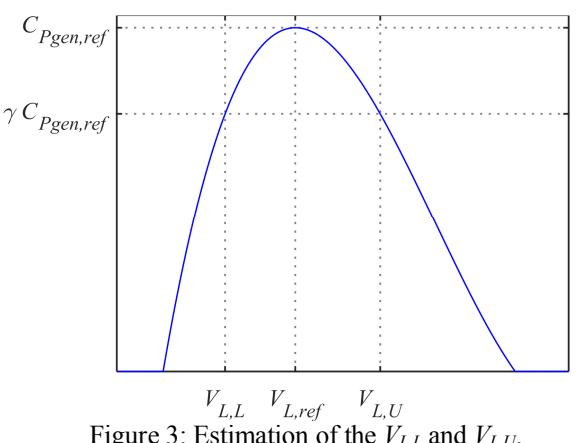

\section{RESULTS \& DISCUSSION}

Performances of MPPT, MPC and SNC algorithms are compared for a standard the wind gust as defined in [22] and the real wind profile reported in [23]. The wind gust profile has a peak amplitude of $5 \mathrm{~m} / \mathrm{s}$ with a period of $10 \mathrm{~s}$. On the other hand, the real wind profile was logged in an urban environment (i.e., on top of a building) for a similar turbine and has very fast dynamics within a large range.

Electromechanical simulations are performed to compare the control algorithms in an idealized software environment and to show that the SNC is an MPC surrogate. HIL experiments are carried out to compare the MPPT and SNC algorithms in a more realistic environment in which inevitable noises and disturbances prevail. In comparisons of controller performance, energy efficiency, $\eta_{E}$, which is the ratio of the energy generated to the maximum available energy, $P_{\max }=C_{P \text { gen,ref }} \rho L R U^{3}$ in the wind for $0 \leq t \leq t_{f}$, is used:

$$
\eta_{E}=\int_{t=0}^{t_{f}} \frac{P_{\text {gen }}}{P_{\max }} d t
$$

\section{A. Electromechanical Simulations}

First, MPPT, MPC and SNC algorithms are compared for the wind gust profile in Fig. $4 \mathrm{a}$, and the responses of $\omega, I_{L}, V_{L}$ and $P_{\text {gen }}$ are shown in Figs. $4 \mathrm{~b}-\mathrm{e}$ respectively. Model-based MPC and SNC methods drive the plant to the reference values, as anticipated, whereas the model-free MPPT does not operate the system at the reference conditions; however, all controllers are able to maximize the $P_{\text {gen }}$ without a considerable steady-state error. In addition, the settling time is roughly the same for all controllers showing that they are tuned properly. Moreover, the responses of the MPC and the SNC as well as their resulting energy efficiencies throughout the 40 -second simulation (i.e., $82.34 \%$ and $81.79 \%$, respectively) are very close. On the other hand, the MPPT harvests slightly lower, $79.43 \%$ of the energy available in the wind.

Second, the performances of the controllers are compared for the real wind profile in Fig. 5a. As in the step wind and gust profiles (Figs. 2 and 4), the responses of the MPC and the SNC are almost indistinguishable and their energy efficiencies are very close, $97.32 \%$ and $97.24 \%$, respectively. Thus, SNC serves as an effective surrogate for the MPC. Furthermore, although there is not an apparent discrepancy between the power outputs (see Fig. 5e), the MPC and SNC algorithms outperform the MPPT method which yields an efficiency of $95.54 \%$. This result confirms that maximizing the instantaneous power does not warrant maximum energy output.

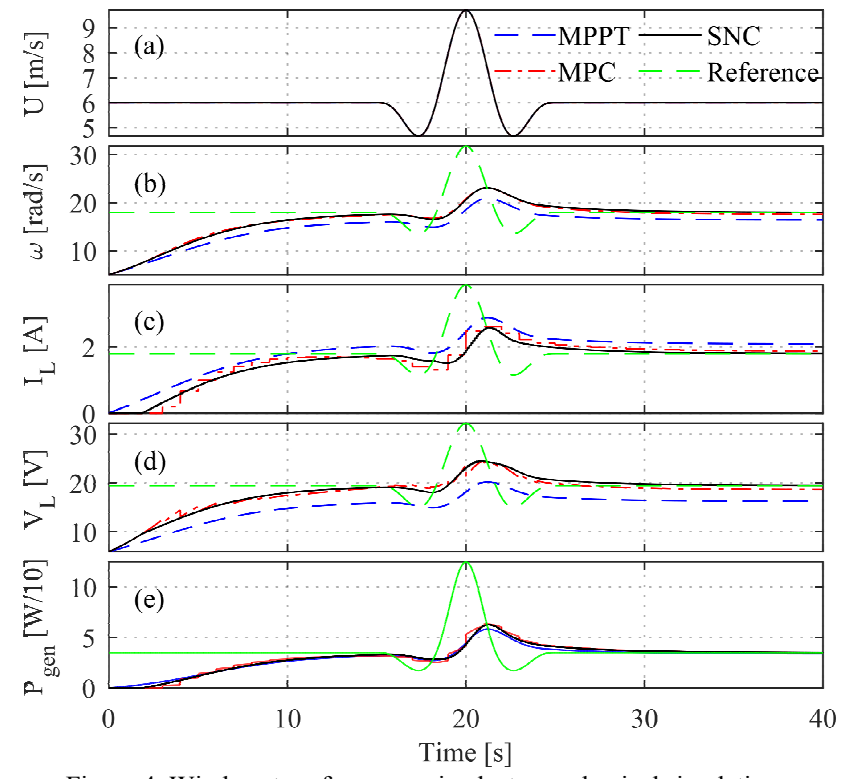

Figure 4: Wind gust performances in electromechanical simulations.

\section{B. Hardware-in-the-loop Simulations}

HIL simulations are carried out to test the MPPT and SNC algorithms for the same wind conditions in real-time with actual electromechanical and power electronics components except the rotor. Fig. 6 demonstrates the $\omega, I_{L}, V_{L}$ and $P_{\text {gen }}$ responses for the wind gust profile in Fig. 6a. Both controllers are observed to perform successfully in the HIL experiments as well. Nonetheless, there is relatively larger steady-state error for the $P_{\text {gen }}$ with the MPPT, and the $P_{g e n}$ for the SNC reaches to greater values, albeit slightly, during the gust than for the MPPT. Overall, the SNC harvests $94.34 \%$ of the available energy while the MPPT harvests $85.20 \%$. 


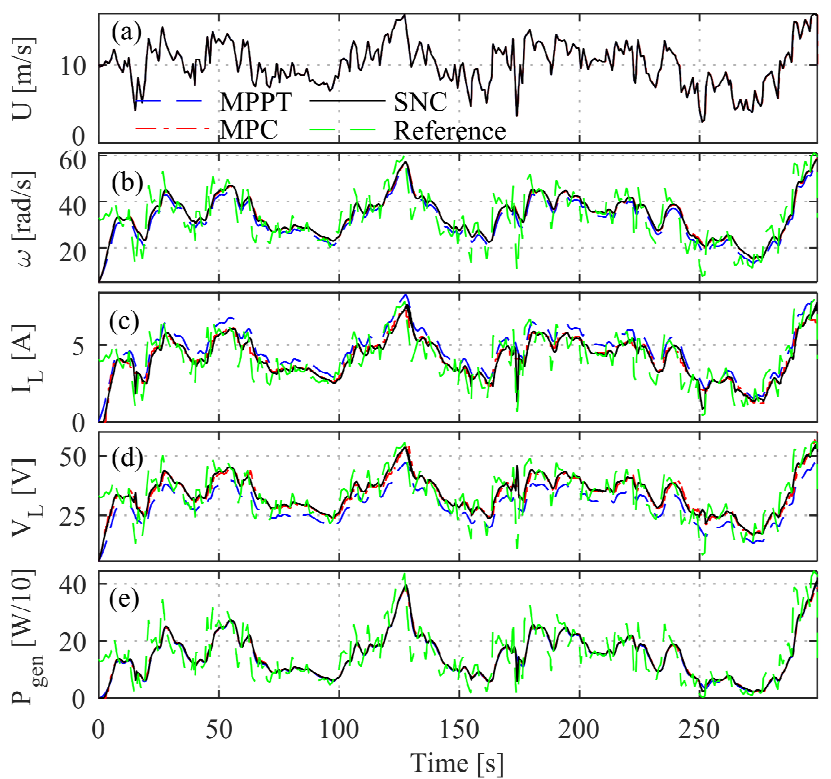

Figure 5: Real wind performances in electromechanical simulations.
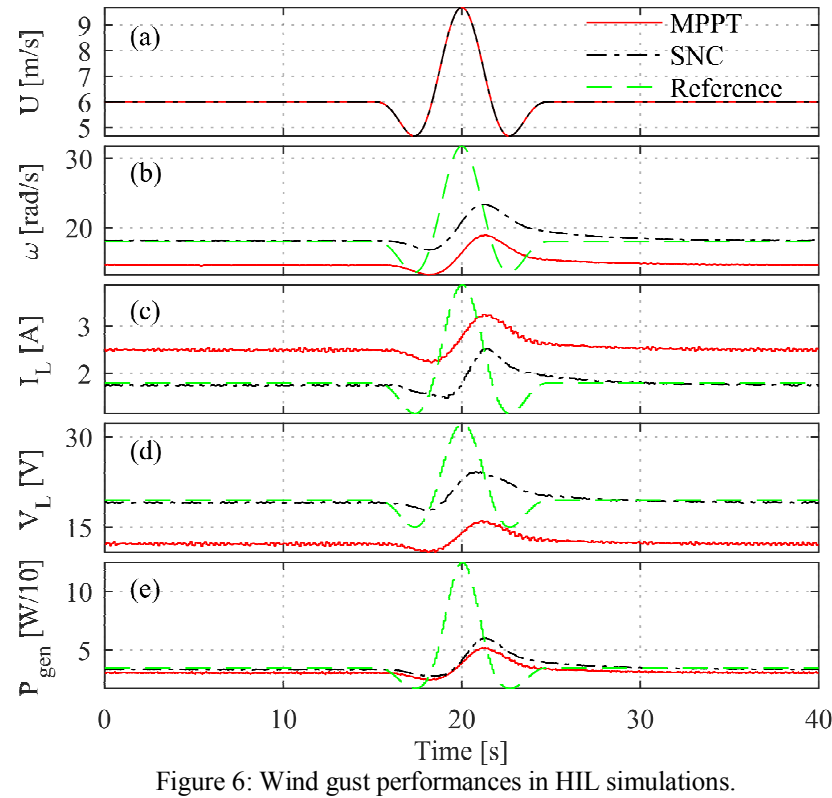

The MPPT and SNC methods are compared for the real wind profile as shown in Fig. 7a. In this case, it is seen that the $\omega$ saturates at about $40 \mathrm{rad} / \mathrm{s}$ (see Fig. 7b), which is the maximum limit for the rotor velocity for HIL simulations because the maximum velocity of the electrical motor is 4000 $\mathrm{rpm}$, which corresponds to about $400 \mathrm{rpm}(41.9 \mathrm{rad} / \mathrm{s})$ at the generator side; furthermore, this limitation saturates the $I_{L}, V_{L}$ and $P_{\text {gen }}$ variables as well, as seen in Figs. $7 \mathrm{c}, \mathrm{d}$, and e. Since the MPPT operates the system at higher current - lower voltage conditions than the reference conditions, the $\omega$, which is correlated with the voltage, is generally lower for the MPPT than the SNC; therefore, the SNC is affected by these saturations more than the MPPT. The $P_{\text {gen }}$ for the MPPT controller exceeds the $P_{\text {gen }}$ for the SNC during the saturation occasions (e.g., for $120<t<150 \mathrm{~s}$ ), as shown in Figure 7e. In addition, for the SNC, there are certain fluctuations in the $I_{L}$ as well as in the resulting $V_{L}$ and $P_{\text {gen }}$ (see Figs. 7b, c, and d) particular- ly when the $U$ changes rapidly, e.g., for $150<t<175$ s. At the end of the experiments, the MPPT and SNC harvest $90.52 \%$ and $90.04 \%$ of the available energy, respectively. In accordance with this outcome, one can suggest that the differences between the power maximizing (MPPT) and energy maximizing (SNC) control algorithms are not significant during rapidly varying realistic wind conditions in the long run. Nevertheless, it is obvious that $\omega$ saturation has a consequential impact on the performance of the SNC.

The MPPT technique requires neither a model nor wind velocity measurement, as distinct from the SNC; therefore, it is more practical than the SNC in application. Moreover, the operation of the MPPT is almost as stable and efficient as the SNC. Thus, although the SNC is found to be a slightly more energy-efficient, the MPPT is an attractive option for smallscale VAWT applications.

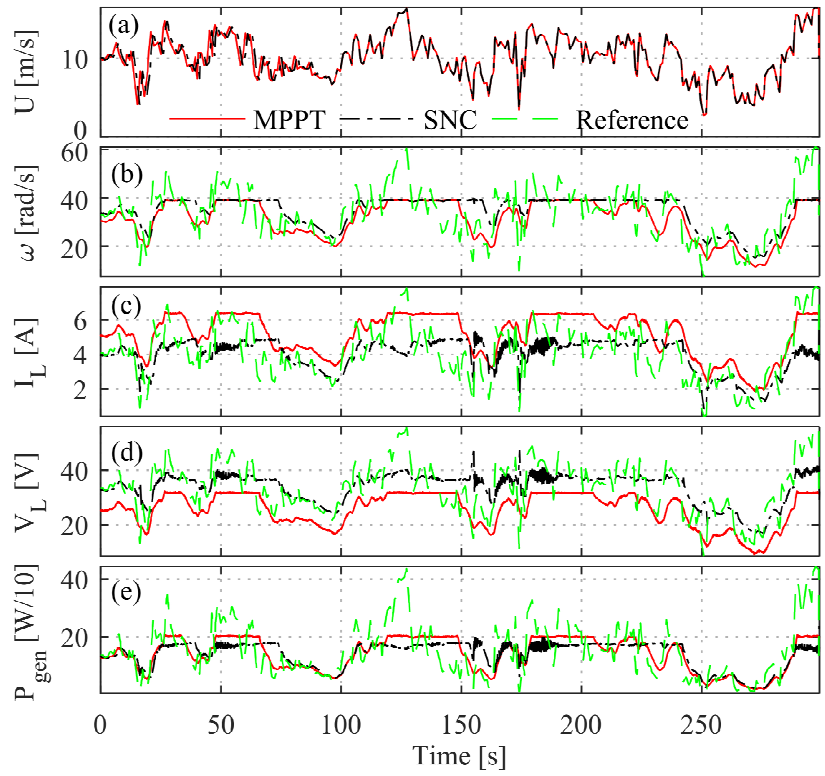

Figure 7: Real wind performances in HIL simulations.

\section{Effect of Power Coefficient Oscillations}

Lastly, the impact of $C_{P}$ oscillations is analyzed through an electromechanical simulation, in which the $C_{P}(\lambda, t)$ is calculated as given in (3) as differently from previous cases in which only the average value of the power coefficient is taken into consideration. This investigation is conducted only for the wind gust profile and by employing the SNC, and the results are depicted in Fig. 8. According to Fig. 8, there is not a visible disparity between the responses and the energy efficiency is effectively unchanged $81.81 \%$ vs $81.79 \%$ when the $C_{P}$ oscillations are taken into account. Thus, it can be concluded that the oscillations in the $C_{P}$ that are caused by the three-straight-bladed structure of the rotor have not a considerable influence on the energy output of the system. Nevertheless, for rotor configurations with a smaller inertia, and slower rotations of the rotor, oscillations in the power output may affect the performance. Further studies would be useful to investigate the role of the inertia of the rotor and the controller design in the suppression of torque ripples and the maximization of the energy output. 


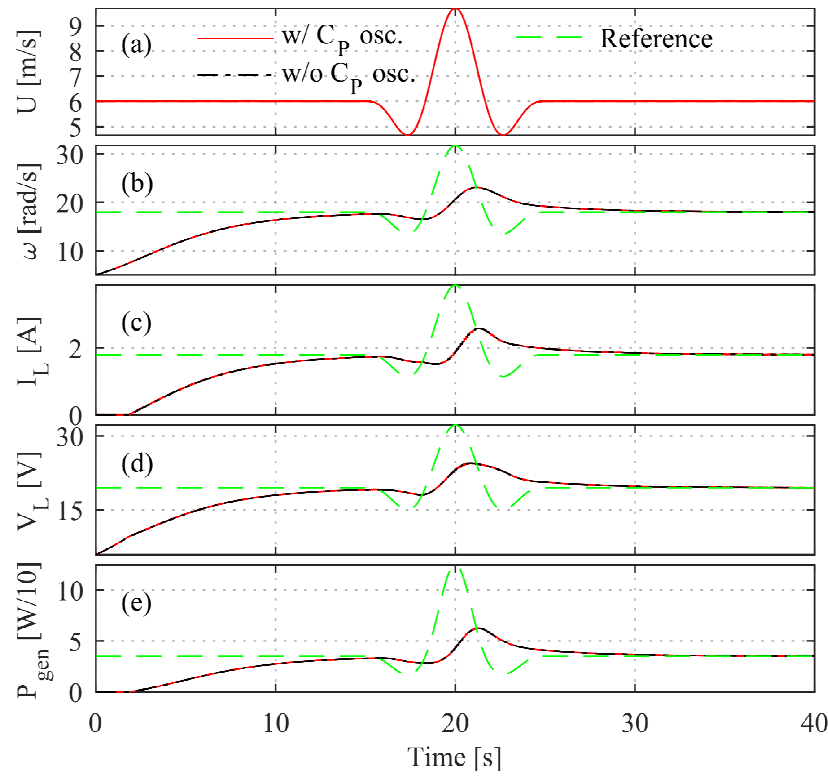

Figure 8: Effect of $C_{P}$ oscillations in electromechanical simulations.

\section{CONCLUSION}

In this study, power-maximizing (HCS-MPPT) and energy maximizing (MPC and SNC) control algorithms are developed and compared. HCS-MPPT is a model-free and sensorless method, whereas MPC and SNC rely on accurate $C_{P^{-}}$ $\lambda$ curves of the VAWT and wind measurements. The SNC is designed as a real-time surrogate for the MPC. In addition, an electromechanical model, which is the simplified DC equivalent of the HIL test-bed, is developed and then used for the design and performance evaluation of the controllers. The proposed controllers are tested for a standardized wind gust and a real wind profile through both the electromechanical model and the HIL test-bed. It is shown that the SNC serves as a surrogate for the MPC, and the MPPT and SNC methods perform successfully in all cases. Although the SNC is found to be slightly more efficient than the MPPT, the reliable and efficient operation of the model-free and sensorless MPPT makes it an attractive option for small-scale applications. Lastly, transient oscillations in the power coefficient are represented with a simple model and used in an electromechanical simulation for the wind gust profile. Results show that the wind torque oscillations due to the three-straight-bladed structure are filtered out by the inertia of the rotor and have negligible effect on the energy-output.

\section{REFERENCES}

[1] Tummala, A., Velamati, R. K., Sinha, D. K., Indraja, V., \& Krishna, V. H. (2016). A review on small scale wind turbines. Renewable and Sustainable Energy Reviews, 56, 1351-1371.

[2] Riegler, H. (2003). HAWT versus VAWT: Small VAWTs find a clear niche. Refocus, 4(4), 44-46.

[3] Pope, K., Dincer, I., \& Naterer, G. F. (2010). Energy and exergy efficiency comparison of horizontal and vertical axis wind turbines. Renewable Energy, 35(9), 2102-2113.

[4] Sutherland, H. J., Berg, D. E., \& Ashwill, T. D. (2012). A retrospective of VAWT technology. SAND2012-0304. Sandia National Laboratories.

[5] Bhutta, M. M. A., Hayat, N., Farooq, A. U., Ali, Z., Jamil, S. R., \& Hussain, Z. (2012). Vertical axis wind turbine-A review of various configurations and design techniques. Renewable and Sustainable Energy Reviews, 16(4), 1926-1939.

[6] Ishugah, T. F., Li, Y., Wang, R. Z., \& Kiplagat, J. K. (2014). Advances in wind energy resource exploitation in urban environment: A review. Renewable and Sustainable Energy Reviews, 37, 613-626.

[7] Salle, S. D. L., Reardon, D., Leithead, W. E., \& Grimble, M. J. (1990). Review of wind turbine control. International Journal of Control, 52(6), 1295-1310.

[8] Johnson, K. E., Pao, L. Y., Balas, M. J., \& Fingersh, L. J. (2006). Control of variable-speed wind turbines: standard and adaptive techniques for maximizing energy capture. IEEE Control Systems, 26(3), 70-81.

[9] Da Rosa, A.V. (2013). Fundamentals of Renewable Energy Processes, Academic Press.

[10] Abdullah, M. A., Yatim, A. H. M., Tan, C. W., \& Saidur, R. (2012). A review of maximum power point tracking algorithms for wind energy systems. Renewable and Sustainable Energy Reviews, 16(5), 32203227.

[11] Koutroulis, E., \& Kalaitzakis, K. (2006). Design of a maximum power tracking system for wind-energy-conversion applications. IEEE Transactions on Industrial Electronics, 53(2), 486-494.

[12] Onol, A. O., Sancar, U., Onat, A., \& Yesilyurt, S. (2015, October). Model Predictive Control for Energy Maximization of Small Vertical Axis Wind Turbines. In ASME 2015 Dynamic Systems and Control Conference. ASME.

[13] Dang, D. Q., Wu, S., Wang, Y., \& Cai, W. (2010, October). Model predictive control for maximum power capture of variable speed wind turbines. In IPEC, 2010 Conference Proceedings (pp. 274-279). IEEE.

[14] Mirzaei, M., Kj, N., \& Niemann, H. H. (2012, June). Robust model predictive control of a wind turbine. In 2012 American Control Conference (ACC) (pp. 4393-4398). IEEE.

[15] Hovgaard, T. G., Boyd, S., \& Jørgensen, J. B. (2015). Model predictive control for wind power gradients. Wind Energy, 18(6), 991-1006.

[16] Henriksen, L. C., Hansen, M. H., \& Poulsen, N. K. (2012). Wind turbine control with constraint handling: a model predictive control approach. IET Control Theory \& Applications, 6(11), 1722-1734.

[17] Onol, A. O., \& Yesilyurt, S. (2017). Effects of wind gusts on a vertical axis wind turbine with high solidity. Journal of Wind Engineering and Industrial Aerodynamics, 162, 1-11. (Accepted/In Press)

[18] Sancar, U., Onol, A.O., Onat, A., \& Yesilyurt, S. (2015, November). Hardware-in-the-loop simulations and control design for small vertical axis wind turbines. In 2015 XXV International Conference on Information, Communication and Automation Technologies. IEEE.

[19] Sancar, U. (2015). Hardware-in-the-loop Simulations and Control Designs for a Vertical Axis Wind Turbine (Master's thesis). Sabanci University.

[20] Scheurich, F., \& Brown, R. E. (2013). Modelling the aerodynamics of vertical-axis wind turbines in unsteady wind conditions. Wind Energy, 16(1), 91-107.

[21] Bausas, M.D., Danao, L.A.M. (2015). The aerodynamics of a camberbladed vertical axis wind turbine in unsteady wind. Energy, 93, 11551164.

[22] International Electrotechnical Commission. (2005). IEC 61400-1: Wind turbines part 1: Design requirements. International Electrotechnical Commission.

[23] Kooiman, S., \& Tullis, S. (2010). Response of a vertical axis wind turbine to time varying wind conditions found within the urban environment. Wind Engineering, 34(4), 389-402. 